\title{
WHAT DO COMPOSITION OPERATORS KNOW ABOUT INNER FUNCTIONS?
}

\author{
JOEL H. SHAPIRO
}

\begin{abstract}
This paper gives several different ways in which operator norms characterize those composition operators $C_{\varphi}$ that arise from holomorphic self-maps $\varphi$ of the unit disc that are inner functions. The setting is the Hardy space $H^{2}$ of the disc, and the key result is a characterization of inner functions in terms of the asymptotic behavior of the Nevanlinna counting function.
\end{abstract}

\section{INTRODUCTION}

Our setting is the Hardy space $H^{2}$; all those functions holomorphic in the open unit disc $\mathbb{U}$ whose Taylor expansion about the origin has square summable coefficient sequence. $H^{2}$ is, in the obvious norm, a Hilbert space - one often viewed as the most natural setting in which to study the interaction between operator theory and complex function theory.

The objects of study here are operators induced on $H^{2}$ by composition with holomorphic self-maps of $\mathbb{U}$. More precisely, given a holomorphic function $\varphi: \mathbb{U} \rightarrow \mathbb{U}$ we define the composition operator $C_{\varphi}$ on the space of all functions holomorphic on $\mathbb{U}$ by:

$$
C_{\varphi} f=f \circ \varphi \quad(f \text { holomorphic on } \mathbb{U}) .
$$

It is obvious that $C_{\varphi}$ is linear, but what is not obvious is that it takes $H^{2}$ into itself. This is the qualitative content of Littlewood's Subordination Principle, which also shows that $C_{\varphi}$ is a bounded operator on $H^{2}$ (see [19], [4], [5], or the primary source [11] for further details). Within the past thirty years or so there has evolved a lively enterprise seeking to relate the function-theoretic properties of the holomorphic map $\varphi$ with the operator-theoretic properties of $C_{\varphi}$, with most of the effort concentrated on notions of compactness, cyclicity, and spectra. The monographs [4] and [19] serve as introductions to much of this

Date: June 15, 1999.

Key words and phrases. Composition operator, inner function, essential norm. This work was supported in part by the National Science Foundation. 
work, while the conference proceedings [8] survey some more recent developments.

This paper asks for properties of composition operators that characterize those self-maps of $\mathbb{U}$ that are inner, i.e. have radial limits of modulus one at almost every point of $\partial \mathbb{U}$. Our goal is to find out how the extremal nature of "innerness" translates into operator-theoretic terms.

More than thirty years ago Nordgren proved the first result along these lines, showing that $C_{\varphi}$ is an isometry of $H^{2}$ into itself if and only if $\varphi$ is inner and fixes the origin [14]. In the same paper Nordgren also showed that:

$$
\varphi \text { inner } \Longrightarrow \quad\left\|C_{\varphi}\right\|=\sqrt{\frac{1+|\varphi(0)|}{1-|\varphi(0)|}} .
$$

In this regard we should note that much earlier work of Littlewood implies

$$
\left\|C_{\varphi}\right\| \leq \sqrt{\frac{1+|\varphi(0)|}{1-|\varphi(0)|}}
$$

for any composition operator $C_{\varphi}$ on $H^{2}$ (see, for example, [19, Chapter 1, page 16] for the details).

Later, in the course of investigating the compactness question for composition operators [18], I proved that if $\varphi$ is inner then the essential norm of $C_{\varphi}$ (its distance, in the operator norm, to the subspace of compact operators on $H^{2}$ ) is the same as its norm, as given by the formula in (1) above. The first goal of this note is to prove the converse (Theorem 4.1 below):

$$
\text { If the essential norm of } C_{\varphi} \text { is } \sqrt{\frac{1+|\varphi(0)|}{1-|\varphi(0)|}} \text { then } \varphi \text { is inner. }
$$

Thus the essential norm recognizes whether or not $\varphi$ is inner.

The method of proof hinges on an apparently new characterization of inner functions in terms of the asymptotic properties of the Nevanlinna counting function (Theorem 3.2). As an additional bonus, this result leads to interesting characterizations of inner functions in terms of the norms of the associated composition operators.

At first glance it might seem impossible that the operator norm could characterize inner functions. Note, for example, that in the special case $\varphi(0)=0$ every composition operator, inner-induced or not, has norm one; the estimate (2) above shows that the norm is $\leq 1$, and equality comes from the fact that composition operators fix constant functions. However if one removes the constant functions from consideration by restricting $C_{\varphi}$ to the subspace $H_{0}^{2}$ of functions in $H^{2}$ that vanish at the origin, then the operator norm does the job (Theorem 5.1): 
If $\varphi(0)=0$ then $\varphi$ is inner if and only if $\left\|\left.C_{\varphi}\right|_{H_{0}^{2}}\right\|=1$.

Here the story takes a surprising twist; the case $\varphi(0)=0$ turns out to be special! We will see (Theorem 5.2) that if $\varphi$ does not fix the origin then the operator norm has the same status as the essential norm in determining innerness:

If $\varphi(0) \neq 0$ then $\varphi$ is inner if and only if $\left\|C_{\varphi}\right\|=\sqrt{\frac{1+|\varphi(0)|}{1-|\varphi(0)|}}$.

For the convenience of the reader I summarize the main results in the table below, where in the last row $N_{\varphi}$ denotes the Nevanlinna counting function (see $\S 2.2$ ).

TABLE 1. Summary of major results, Sections $1-5$

\begin{tabular}{|c|c|c|}
\hline $\begin{array}{c}\text { Condition equivalent to: } \\
\text { " } \varphi \text { is inner" }\end{array}$ & $\begin{array}{c}\text { Further hypothesis } \\
\text { on } \varphi\end{array}$ & Reference \\
\hline \hline$C_{\varphi}$ isometric on $H^{2}$ & $\varphi(0)=0$ & {$[14]$} \\
\hline$\left\|\left.C_{\varphi}\right|_{H_{0}^{2}}\right\|=1$ & $\varphi(0)=0$ & Theorem 5.2 \\
\hline$\left\|C_{\varphi}\right\|=\sqrt{\frac{1+|\varphi(0)|}{1-|\varphi(0)|}}$ & $\varphi(0) \neq 0$ & Theorem 5.1 \\
\hline$\left\|C_{\varphi}\right\|=\sqrt{\frac{1+|\varphi(0)|}{1-|\varphi(0)|}}$ & None & Theorem 4.1 \\
\hline $\limsup _{|w| \rightarrow 1-} \frac{N_{\varphi}(w)}{\log \frac{1}{|w|}}=\frac{1+|\varphi(0)|}{1-|\varphi(0)|}$ & None & Theorem 3.2 \\
\hline
\end{tabular}

The next section outlines the prerequisites, while those that follow characterize inner functions in terms of successively: the asymptotics of the Nevanlinna counting function, the essential operator norm, and the operator norm. The paper closes with some final remarks describing further extensions of the main results and connections with some other work in the literature.

\section{Prerequisites}

This section collects, mostly without proof, known results that are needed for the sequel. 
2.1. The $H^{2}$-norm. Recall that $H^{2}$ is the set of functions $f(z)=$ $\sum_{n=0}^{\infty} \hat{f}(n) z^{n}$ holomorphic in the unit disc, with $\sum_{n=0}^{\infty}|\hat{f}(n)|^{2}<\infty$. The natural norm $\|\cdot\|$ defined by

$$
\|f\|^{2}=\sum_{n=0}^{\infty}|\hat{f}(n)|^{2} \quad\left(f \in H^{2}\right)
$$

turns $H^{2}$ into a Hilbert space.

The norm of a function $f \in H^{2}$ can be calculated in terms of area integrals by the Littlewood-Paley identity.

$$
\|f\|^{2}=|f(0)|^{2}+2 \int_{\mathbb{U}}\left|f^{\prime}(z)\right|^{2} \log \frac{1}{|z|} d A(z) \quad\left(f \in H^{2}\right),
$$

where $d A$ denotes Lebesgue area measure on $\mathbb{U}$, normalized to have unit mass. This result is easily established by substituting the power series representation for $f$ into the right-hand side of (4); after some standard manipulations this reduces to the right-hand side of (3) (see $[19, \S 3.1$ and $\S 3.7$, Exercise 1] for more details).

2.2. The Change-of-Variable Formula. For any holomorphic selfmap $\varphi$ of $\mathbb{U}$ and any $f \in H^{2}$, a careful application of the usual changeof-variable formula for multiple integrals to the right-hand side of (4) leads to this:

$$
\left\|C_{\varphi} f\right\|^{2}=|f(\varphi(0))|^{2}+2 \int_{\mathbb{U}}\left|f^{\prime}\right|^{2} N_{\varphi} d A,
$$

where $N_{\varphi}$ is the Nevanlinna counting function, defined on $\varphi(\mathbb{U}) \backslash\{\varphi(0)\}$ by:

$$
N_{\varphi}(w)=\sum_{z \in \varphi^{-1}\{w\}} \log \frac{1}{|z|},
$$

wherein points of inverse image $\varphi^{-1}\{w\}$ are regarded as being repeated according to their $\varphi$-multiplicity. If $w \notin \varphi(\mathbb{U})$ then the sum on the right is "empty" and $N_{\varphi}(w)$ is defined to be zero. The only point of the unit disc left in question is $w=\varphi(0)$, at which it is natural to give $N_{\varphi}$ the value $+\infty$, a choice further legitimized by the fact that $N_{\varphi}$ has a logarithmic pole at $\varphi(0)$; see $[19, \S 10.1, \S 10.3]$ and $\S 3.3$ for more details.

Formula (5) is a special case of a more general (and spectacularly useful) one due to C.S. Stanton; for more on Stanton's remarkable formula see [6, Theorem 2, page 130], and the primary reference, [21, Chapter 1]. 
2.3. Standard automorphisms. For each point $w$ of $\mathbb{U}$ we define

$$
\alpha_{w}(z) \stackrel{\text { def }}{=} \frac{w-z}{1-\bar{w} z} \quad(z \in \mathbb{U}) .
$$

It is elementary to check that the linear-fractional transformation $\alpha_{w}$ is a conformal automorphism of $\mathbb{U}$ that interchanges $w$ with the origin, and is its own inverse.

Of great utility is the following fundamental identity involving these "standard automorphisms:"

$$
1-\left|\alpha_{w}(z)\right|^{2}=\frac{\left(1-|w|^{2}\right)\left(1-|z|^{2}\right)}{|1-\bar{w} z|^{2}} \quad(z \in \mathbb{U})
$$

(see $[19, \S 4.3$, pp. 59-60] or [7, page 3]). Our first application of this identity is to the proof a lemma that will figure importantly in everything that follows.

2.4. Lemma. If $E$ is any subset of $\mathbb{U}$ whose closure contains $\partial \mathbb{U}$, then

$$
\limsup _{|w| \rightarrow 1-, w \in E} \frac{\log \left|\alpha_{w}(p)\right|}{\log |w|}=\frac{1+|p|}{1-|p|}
$$

for any $p \in \mathbb{U}$.

Proof. If $p=0$ there is nothing to prove, since $\alpha_{w}(0)=w$; so suppose $p \neq 0$. Now $-\log x \approx 1-x$ as $x \rightarrow 1-$ (meaning: $\frac{-\log x}{1-x} \rightarrow 1$ as $x \rightarrow 1-)$. We see from (6) that $\left|\alpha_{w}(p)\right| \rightarrow 1$ as $|w| \rightarrow 1-$, so

$$
\frac{\log \left|\alpha_{w}(p)\right|}{\log |w|}=\frac{\log \left(\left|\alpha_{w}(p)\right|^{2}\right)}{\log \left(|w|^{2}\right)} \approx \frac{1-\left|\alpha_{w}(p)\right|^{2}}{1-|w|^{2}}=\frac{1-|p|^{2}}{|1-\bar{w} p|^{2}},
$$

where the last equality comes from the identity (6). Clearly the quantity on the right-hand side of (7) is $\leq \frac{1+|p|}{1-|p|}$. Further, because the set $E$ contains $\partial \mathbb{U}$ in its closure, there exists a sequence $\left(w_{n}\right)$ of points in $E$ that converges to $p /|p|$ (recall that $p \neq 0)$. For this sequence, (7) guarantees that

$$
\frac{\log \left|\alpha_{w_{n}}(p)\right|}{\log \left|w_{n}\right|} \rightarrow \frac{1-|p|^{2}}{(1-|p|)^{2}}=\frac{1+|p|}{1-|p|},
$$

which finishes the argument.

2.5. Frostman Transforms. For each holomorphic self-map $\varphi$ of $\mathbb{U}$ and each $w \in \mathbb{U}$ we define the Frostman transform of $\varphi$ by $w$ to be $\varphi_{w}=\alpha_{w} \circ \varphi$. Thus $\varphi_{w}$ is also a holomorphic self-map of $\mathbb{U}$ for each $w \in \mathbb{U}$. The terminology comes from a famous theorem of Frostman which asserts that if $\varphi$ is inner then $\varphi_{w}$ is a Blaschke product for all $w \in \mathbb{U}$ with exception of a (possibly empty) set of logarithmic capacity 
zero, i.e. for "quasi-every" (abbreviated "q.e.") $w \in \mathbb{U} .{ }^{1}$ For a proof see, for example, [7, Theorem 6.8, page 79]. Rudin [16] has proved a useful generalization of Frostman's theorem which implies that: If $\varphi$ is any holomorphic self-map of $\mathbb{U}$ then $\varphi_{w}$ has no singular inner factor for q.e. $w \in \mathbb{U}$.

2.6. Properties of the counting function. While the results of this section are well known, they are hard to find in one place in the literature. For a detailed exposition, along with proofs, I invite the reader to download the expository article [20] from my website at http://www . math.msu.edu/ shapiro/preprints/.

A crucial property of the Nevanlinna counting function for the study of composition operators is Littlewood's Inequality, which states that for any holomorphic self-map of $\mathbb{U}$,

$$
N_{\varphi}(w) \leq \log \frac{1}{\left|\varphi_{w}(0)\right|}=\log \left|\frac{1-\bar{w} \varphi(0)}{w-\varphi(0)}\right| \quad(w \in \mathbb{U}) .
$$

For a proof see $[19, \S 10.4],[18, \S 4.2],[20, \S 2.3]$, or the next few paragraphs.

In case $\varphi(0)=0$ the right-hand side of Littlewood's inequality is just $\log (1 /|w|)$, which expresses the fact that the counting function decays to zero like the distance to the boundary, and, in concert with the Littlewood-Paley identity (4), makes it obvious that the associated composition operator is a contraction on $H^{2}$. After this, the problem of establishing the bound (2) for the norm of an arbitrary composition operator on $H^{2}$ reduces to that of establishing it for the standard automorphism $\alpha_{\varphi(0)}$ (see [19, page 16] for the details).

The counting function has a subharmonic majorant that will figure importantly in the sequel. Jensen's formula shows that for each $w \in \mathbb{U}$,

$$
N_{\varphi}(w)=\lim _{r \rightarrow 1-} \int_{\partial \mathbb{U}} \log \left|\varphi_{w}(r \zeta)\right| d m(\zeta)+\log \frac{1}{\left|\varphi_{w}(0)\right|},
$$

where $m$ denotes Lebesgue arclength measure on $\partial \mathbb{U}$, normalized to have total mass 1 ([18, $\S 4.2],[19, \S 10.4],[20, \S 2.3])$. This formula makes Littlewood's Inequality (8) obvious; $\left|\varphi_{w}\right|<1$ on $\mathbb{U}$ so the integral on the right-hand side of (9) is negative.

Every $f \in H^{2}$, and in particular, every bounded holomorphic $f$, has a radial limit $f(\zeta)=\lim _{r \rightarrow 1-} f(r \zeta)$ at $m$-a.e. point $\zeta \in \partial \mathbb{U}$. Thus an application of Fatou's Lemma from integration theory to (9) shows

\footnotetext{
${ }^{1}$ For our purposes it is enough to know that "quasi-every ..." implies "almost every ...", i.e. that sets of capacity zero have Lebesgue area measure zero.
} 
that

$$
N_{\varphi}(w) \leq \bar{N}_{\varphi}(w) \stackrel{\text { def }}{=} \int_{\partial \mathbb{U}} \log \left|\varphi_{w}(\zeta)\right| d m(\zeta)+\log \frac{1}{\left|\varphi_{w}(0)\right|}
$$

for each $w \in \mathbb{U}$. The rest of what we need comes from an argument involving Rudin's generalization of Frostman's Theorem, which shows that not only does $\bar{N}_{\varphi}$ dominate $N_{\varphi}$ at each point of $\mathbb{U}$, but the two functions actually coincide q.e. on $\mathbb{U}$ ! (see [18, §4.2], or [20] for details).

Although not necessary for the sequel, it is worth noting at this point that for each $\zeta \in \partial \mathbb{U}$ at which $\varphi$ has a radial limit,

$$
\log \left|\varphi_{w}(\zeta)\right|=\log |w-\varphi(\zeta)|+\log \frac{1}{|1-\bar{w} \varphi(\zeta)|}
$$

is the sum of a subharmonic function of $w$ and a harmonic one, hence $\bar{N}_{\varphi}$ is itself subharmonic in $w$, and therefore $N_{\varphi}$ is subharmonic at quasi-every point of $\mathbb{U}$.

Finally, the work of Section 5 will require the following simple result about integrals of monotonic functions.

2.7. Lemma. Suppose $\mu$ is a positive, finite Borel measure on the interval $[0,1)$ that has the point 1 in its closed support. Then for each $0<r<1$ there exists a constant $0<\gamma=\gamma(\mu, r)<1$ such that

$$
\int_{[0, r)} g d \mu \leq \gamma \int_{[0,1)} g d \mu
$$

for every non-negative monotonically increasing function $g$ on $[0,1)$.

Proof. Let $a=\mu\{[0, r)\}$ and $b=\mu\{[r, 1)\}$. Then $b>0$ by our hypothesis on the support of $\mu$, and we may assume that also $a>0$; otherwise there would be nothing to prove. Fix an increasing, non-negative function $g$ on $[0,1)$. Then:

$$
\begin{aligned}
\int_{[0,1)} g d \mu & =\int_{[0, r)} g d \mu+\int_{[r, 1)} g d \mu \\
& \geq \int_{[0, r)} g d \mu+\mu\{[r, 1)\} g(r) \\
& =\int_{[0, r)} g d \mu+\frac{b}{a} \mu\{[0, r)\} g(r) \\
& \geq\left(1+\frac{b}{a}\right) \int_{[0, r)} g d \mu,
\end{aligned}
$$

which gives the desired result with $\gamma=\left(1+\frac{b}{a}\right)^{-1}$. 


\section{Inner Functions And the Counting Function}

A closer look at the proof outlined above for Littlewood's inequality shows that there is equality for some point of $\mathbb{U}$ if and only if there is equality for quasi-every point, and that this happens precisely when $\varphi$ is an inner function (see $[18, \S 4.2],[20, \S 3.1]$ for the details). Thus inner functions can be recognized by the pointwise behavior of their counting functions.

In this section we consider the question of whether or not inner functions can be recognized by the asymptotic behavior of their counting functions. The precise behavior of $N_{\varphi}$ when $\varphi$ is inner is well known:

\subsection{Lemma $[18, \S 2.5]$. For every inner function $\varphi$ :}

$$
\limsup _{|w| \rightarrow 1-} \frac{N_{\varphi}(w)}{\log \frac{1}{|w|}}=\frac{1+|\varphi(0)|}{1-|\varphi(0)|} .
$$

Proof. Because inner functions achieve equality q.e. in Littlewood's inequality (8), we have, at every point $w$ of a subset $E$ of $\mathbb{U}$ whose complement has capacity zero,

$$
N_{\varphi}(w)=\log \frac{1}{\left|\varphi_{w}(0)\right|}=\log \frac{1}{\left|\alpha_{w}(\varphi(0))\right|} .
$$

Because $E$ is dense in $\mathbb{U}$ it obeys the hypothesis of Lemma 2.4 (with $p=\varphi(0))$, so the desired result (10) follows from that Lemma.

The question remains: If $\varphi$ satisfies (10), is it an inner function? The following result shows that the answer is "yes".

3.2. Theorem. If $\varphi$ is a holomorphic self-map of $\mathbb{U}$ that is not inner, then there exist $0<r, \delta<1$ such that

$$
N_{\varphi}(w) \leq \delta \log \frac{1}{\left|\varphi_{w}(0)\right|} \quad \forall r<|w|<1 .
$$

Proof. Suppose first that $\varphi(0)=0$, so that our goal becomes that of finding $0<\delta, r<1$ such that

$$
N_{\varphi}(w) \leq \delta \log \frac{1}{|w|} \quad \forall r<|w|<1 .
$$

We actually prove this for the larger function $\bar{N}_{\varphi}$.

We are assuming that $\varphi$ is not inner, so there exists a Borel set $E \subset \partial \mathbb{U}$ with $m(E)>0$, and a constant $0<\kappa<1$ such that $|\varphi(\zeta)|<\kappa$ 
for every $\zeta \in E$. Since $\varphi_{w}=\alpha_{w} \circ \varphi$, the fundamental identity (6) implies for every $w \in \mathbb{U}$ and $\zeta \in E$ :

$$
\begin{aligned}
1-\left|\varphi_{w}(\zeta)\right|^{2} & =\frac{\left(1-|w|^{2}\right)\left(1-|\varphi(\zeta)|^{2}\right)}{|1-\bar{w} \varphi(\zeta)|^{2}} \\
& \geq \frac{\left(1-|w|^{2}\right)\left(1-\kappa^{2}\right)}{(1+|\varphi(\zeta)|)^{2}} \\
& \geq \frac{\left(1-|w|^{2}\right)\left(1-\kappa^{2}\right)}{(1+\kappa)^{2}}
\end{aligned}
$$

hence

$$
1-\left|\varphi_{w}(\zeta)\right|^{2} \geq\left(1-|w|^{2}\right) \frac{1-\kappa}{1+\kappa} \quad(\forall \zeta \in E, w \in \mathbb{U}) .
$$

Now $\log x \leq x-1$ for every positive $x$, and for every $x \in(0,1)$ that is sufficiently close to 1 we have $\log x \geq 2(x-1)$. Thus, restricting to $0<x<1$ we have:

$$
1-x \leq \log \frac{1}{x} \leq 2(1-x)
$$

where the first inequality holds for all $x>0$ and the second one for all $x$ in $(0,1)$ close enough to 1 . Upon substituting $x=\left|\varphi_{w}(\zeta)\right|$ in these inequalities and using (13) we obtain for all $w \in \mathbb{U}$ sufficiently large (say $r \leq|w|<1)$ and all $\zeta \in E$ :

$$
\log \frac{1}{\left|\varphi_{w}(\zeta)\right|} \geq \gamma \log \frac{1}{|w|}
$$

where $\gamma \stackrel{\text { def }}{=} \frac{1}{2} \frac{1-\kappa}{1+\kappa}$, a number strictly between 0 and 1 .

Because $\log \left|\varphi_{w}\right| \leq 0$ on $\partial \mathbb{U}$ for every $w \in \mathbb{U}$,

$$
\bar{N}_{\varphi}(w) \stackrel{\text { def }}{=} \int_{\partial \mathbb{U}} \log \left|\varphi_{w}\right| d m+\log \frac{1}{|w|} \leq \int_{E} \log \left|\varphi_{w}\right| d m+\log \frac{1}{|w|},
$$

hence inequality (14) implies that for all $r \leq|w|<1$ :

$$
\bar{N}_{\varphi}(w) \leq-\gamma m(E) \log \frac{1}{|w|}+\log \frac{1}{|w|}=\delta \log \frac{1}{|w|}
$$

where $\delta=1-\gamma m(E)$ lies strictly between 0 and 1 . This is the desired result for the case $\varphi(0)=0$.

In case $\varphi(0)=p \neq 0$, the idea is to apply inequality (12) to $\varphi_{p}=$ $\alpha_{p} \circ \varphi$. Since $\varphi_{p}$ is a holomorphic self-map of $\mathbb{U}$ that fixes the origin, the special case we have just completed shows that $N_{\varphi_{p}}(w) \leq \delta \log \frac{1}{|w|}$ for all $w$ with $r<|w|<1$, where $\delta$ and $r$ are as in the last paragraph. 
The self-inverse property of $\alpha_{p}$ along with the definition of the counting function yields $N_{\varphi}(w)=N_{\varphi_{p}}\left(\alpha_{p}(w)\right)$, so that

$$
N_{\varphi}(w) \leq \delta \log \frac{1}{\left|\alpha_{p}(w)\right|}=\delta \log \frac{1}{\left|\varphi_{w}(0)\right|}
$$

for all $w \in \mathbb{U}$ with $\left|\alpha_{p}(w)\right|>r$. This set of points $w$ is the image of the annulus $\{r<|z|<1\}$ under $\alpha_{p}^{-1}=\alpha_{p}$, so it is an annulus with outer boundary the unit circle and inner boundary a circle in $\mathbb{U}$ centered at $p$. In particular, this "eccentric annulus" contains a centered one $\left\{r^{\prime}<|w|<1\right\}$. Thus (11) holds for every $r^{\prime}<|w|<1$, which completes the proof.

3.3. Remark. The restriction that $w$ lie close to the boundary of $\mathbb{U}$ is necessary because $N_{\varphi}(w)$ behaves like $-\log |w-\varphi(0)|$ as $w \rightarrow \varphi(0)$ (see $[13, \S I I I .4$, page 51] or $[20, \S 2.4]$ ), so no inequality of the form (11) can hold for $w$ sufficiently close to $\varphi(0)$.

\section{Inner Functions and the Essential Norm}

This section reinterprets the result of the previous one in operator theoretic language. Let $\left\|C_{\varphi}\right\|_{e}$ denote the essential norm of the composition operator $C_{\varphi}$; recall that this is the distance, in the norm of operators on $H^{2}$, from $C_{\varphi}$ to subspace of compact operators. The main result of [18] connects the asymptotic behavior of $N_{\varphi}$ with the essential norm of $C_{\varphi}$ via the following formula, valid for every holomorphic self-map $\varphi$ of $\mathbb{U}$ :

$$
\left\|C_{\varphi}\right\|_{e}^{2}=\limsup _{|w| \rightarrow 1-} \frac{N_{\varphi}(w)}{\log \frac{1}{|w|}} .
$$

This formula allows Theorem 3.2 to be reinterpreted as a result about the essential norm.

4.1. Theorem. For a holomorphic self-map $\varphi$ of $\mathbb{U}$, the following conditions are equivalent:

(a) $\varphi$ is inner.

(b) $\left\|C_{\varphi}\right\|_{e}^{2}=\frac{1+|\varphi(0)|}{1-|\varphi(0)|}$.

Proof. (a) $\rightarrow$ (b): This result, originally proved in [18, Theorem 2.5, page 382] (see also [20, §5.3]), follows immediately from (15) and Lemma 3.1 .

(b) $\rightarrow$ (a): Now assume that $\varphi$ is not inner, so that inequality (11) holds at every point $w$ of an annulus $E=\{r<|w|<1\}$. In this case 
(15), Lemma 2.4, and Theorem 3.2 combine to yield

$$
\left\|C_{\varphi}\right\|_{e}^{2} \leq \delta \frac{1+|\varphi(0)|}{1-|\varphi(0)|}
$$

where $\delta$ is the constant $(<1)$ that shows up on the right-hand side of inequality (11).

4.2. Remark. Recall that the norm of a composition operator $C_{\varphi}$ on $H^{2}$ is always $\leq \frac{1+|\varphi(0)|}{1-|\varphi(0)|}$, and that this bound is attained for inner functions. The result just proved shows that if $\varphi$ is inner then $\left\|C_{\varphi}\right\|=$ $\left\|C_{\varphi}\right\|_{e}$. In view of this we might ask if inner functions are characterized by this equality of norm and essential norm for the induced composition operators. Curiously, this is true if $\varphi(0)=0$, but false otherwise.

To see why, recall that if $\varphi(0)=0$ then $\left\|C_{\varphi}\right\|=1$, whereas Theorem 4.1 asserts that $\varphi$ is inner if and only if $\left\|C_{\varphi}\right\|_{e}=1$. On the other hand, work of Cowen [3] shows that for the special maps $\varphi(z)=s z+(1-s)$ with $0<s<1$, the norm of $C_{\varphi}$ is $1 / \sqrt{s}$, which by formula (15) coincides with the essential norm (see, e.g., $[2, \S 3]$ for the details of this essential norm calculation).

\section{InNer Functions AND the Operator Norm}

Can one decide if $\varphi$ is inner by looking at the operator norm of $C_{\varphi}$ ? The answer is: "Yes and No."

"No" has surfaced several times already in the observation that, regardless of whether or not $\varphi$ is inner, if it fixes the origin then the composition operator it induces has norm one. The point of this section is to exhibit two ways in which the answer is "Yes". The first of these covers the case where $\varphi$ fixes the origin. For this, recall that $H_{0}^{2}$ denotes the subspace of functions in $H^{2}$ that vanish at the origin.

5.1. Theorem. Suppose $\varphi$ is a holomorphic self-map of $\mathbb{U}$ with $\varphi(0)=$ 0 . Then $\varphi$ is inner if and only if the restriction of $C_{\varphi}$ to $H_{0}^{2}$ has norm equal to 1.

Proof. If $\varphi$ is inner then, as mentioned in the Introduction, $C_{\varphi}$ is an isometry o $H^{2}$, hence also on $H_{0}^{2}$, so in particular its restriction to $H_{0}^{2}$ has norm 1.

For the converse, suppose $\varphi$ is not inner. Our goal is to show that the restriction of $C_{\varphi}$ to $H_{0}^{2}$ has norm $<1$. To this end recall that Theorem 3.2 provides numbers $0<r, \delta<1$ such that (12) holds. This, along with the change of variable formula (5) and Littlewood's Inequality (8), 
yields the following estimate, valid for any $f \in H_{0}^{2}$ :

$$
\begin{aligned}
\left\|C_{\varphi} f\right\|^{2} & =2 \int_{\mathbb{U}}\left|f^{\prime}(w)\right|^{2} N_{\varphi}(w) d A(w) \\
& \leq\left(2 \int_{r \mathbb{U}}+2 \delta \int_{\mathbb{U} \backslash r \mathbb{U}}\right)\left|f^{\prime}(w)\right|^{2} \log \frac{1}{|w|} d A(w),
\end{aligned}
$$

which, upon rearrangment of the integrals in the last line, becomes

$$
\left\|C_{\varphi}\right\|^{2} \leq\left(2(1-\delta) \int_{r \mathbb{U}}+2 \delta \int_{\mathbb{U}}\right)\left|f^{\prime}(w)\right|^{2} \log \frac{1}{|w|} d A(w) .
$$

The idea now is to use Lemma 2.7 to estimate the first integral on the right. For this note that, because $\left|f^{\prime}\right|^{2}$ is subharmonic on $\mathbb{U}$, the angular average

$$
g(r) \stackrel{\text { def }}{=} \frac{1}{2 \pi} \int_{0}^{2 \pi}\left|f^{\prime}\left(r e^{i \theta}\right)\right|^{2} d \theta
$$

is monotone increasing for $0 \leq r<1$, and note further that if we set $d \mu(t)=4 \log \frac{1}{t} d t$ then for each $0 \leq \rho \leq 1$ :

$$
\int_{[0, \rho)} g d \mu=\int_{\rho \mathbb{U}}\left|f^{\prime}(w)\right|^{2} \log \frac{1}{|w|} d A(w) .
$$

Thus Lemma 2.7 provides a constant $0<\gamma<1$ that depends only on the previously fixed constant $r$ (in particular, not on $f$ ) such that

$$
\int_{r \mathbb{U}}\left|f^{\prime}(w)\right|^{2} \log \frac{1}{|w|} d A(w) \leq \gamma \int_{\mathbb{U}}\left|f^{\prime}(w)\right|^{2} \log \frac{1}{|w|} d A(w)
$$

Upon substituting this into (16), and then using the Littlewood-Paley identity (4) we obtain:

$$
\left\|C_{\varphi} f\right\|^{2} \leq \gamma(1-\delta)\|f\|^{2}+\delta\|f\|^{2}=\nu\|f\|^{2},
$$

where $\nu=\gamma(1-\delta)+\delta<1$. Since this last inequality holds for each $f \in H_{0}^{2}$, it yields the desired result: The restriction of $C_{\varphi}$ to $H_{0}^{2}$ has norm $\leq \nu<1$.

The next result reiterates a theme first encountered in Remark 4.2: The case $\varphi(0)=0$ is special!

5.2. Theorem. Suppose $\varphi$ is a holomorphic self-map of $\mathbb{U}$ with $\varphi(0) \neq$ 0. Then $\varphi$ is inner if and only if $\left\|C_{\varphi}\right\|=\sqrt{\frac{1+|\varphi(0)|}{1-|\varphi(0)|}}$.

Proof. For $\varphi$ inner the norm calculation was noted in the Introduction; it is due to Nordgren. For the converse, suppose $\varphi$ is not inner. Let $p=\varphi(0) \neq 0$. We want to show that the norm of $C_{\varphi}$ is strictly less than $\sqrt{\frac{1+|p|}{1-|p|}}$. 
For this recall once again the standard automorphism $\alpha_{p}$ defined in $\S 2.3$ and the Frostman transform $\varphi_{p}=\alpha_{p} \circ \varphi$, which in this case vanishes at the origin. Because $\alpha_{p}$ is self-inverse, $\varphi=\alpha_{p} \circ \varphi_{p}$, so we have for every $f \in H^{2}$ :

$$
C_{\varphi} f=C_{\varphi_{p}}\left(f \circ \alpha_{p}\right)=C_{\varphi_{p}} g+f(p)
$$

where $g=f \circ \alpha_{p}-f(p)$. Because $g$ and $\varphi_{p}$ both vanish at the origin, so does $C_{\varphi_{p}}$ g, which is therefore orthogonal in $H^{2}$ to the constant functions. Furthermore, by Theorem 5.1 we know that the restriction of $C_{\varphi_{p}}$ to $H_{0}^{2}$ has norm strictly less than 1 ; let us denote this norm by $\sqrt{\nu}$. From this, (17), and the above-mentioned orthogonality we obtain:

$$
\left\|C_{\varphi} f\right\|^{2}=\left\|C_{\varphi_{p}} g\right\|^{2}+|f(p)|^{2} \leq \nu\|g\|^{2}+|f(p)|^{2},
$$

which we summarize as:

$$
\left\|C_{\varphi} f\right\|^{2} \leq \nu\left\|\left(C_{\alpha_{p}} f\right)-f(p)\right\|^{2}+|f(p)|^{2} .
$$

To continue the calculation observe that, because $\langle h, 1\rangle=h(0)$ for every $h \in H^{2}$,

$$
\left\langle C_{\alpha_{p}} f, f(p)\right\rangle=\overline{f(p)}\left(C_{\alpha_{p}} f(0)\right)=\overline{f(p)} f(p)=|f(p)|^{2},
$$

SO

$$
\begin{aligned}
\left\|\left(C_{\alpha_{p}} f\right)-f(p)\right\|^{2} & =\left\|C_{\alpha_{p}} f\right\|^{2}-2 \operatorname{Re}\left\langle C_{\alpha_{p}} f, f(p)\right\rangle+|f(p)|^{2} \\
& =\left\|C_{\alpha_{p}} f\right\|^{2}-2|f(p)|^{2}+|f(p)|^{2} \\
& =\left\|C_{\alpha_{p}} f\right\|^{2}-|f(p)|^{2} .
\end{aligned}
$$

This last identity along with estimate (18) yields

$$
\left\|C_{\varphi} f\right\|^{2} \leq \nu\left\|C_{\alpha_{p}} f\right\|^{2}+(1-\nu)|f(p)|^{2} .
$$

Now $f(p)=\left\langle f, K_{p}\right\rangle$, where $K_{p}(z)=(1-\bar{p} z)^{-1}$, so

$$
|f(p)| \leq\|f\|\left\|K_{p}\right\|=\frac{\|f\|}{\sqrt{1-|p|^{2}}},
$$

and we know that $\left\|C_{\alpha_{p}}\right\|^{2} \leq \frac{1+|p|}{1-|p|}$ (actually there is equality here; see (1)). Upon using these results in the right-hand side of (19) we obtain:

$$
\begin{aligned}
\left\|C_{\varphi} f\right\|^{2} & \leq \nu\left(\frac{1+|p|}{1-|p|}\right)\|f\|^{2}+\left(\frac{1-\nu}{1-|p|^{2}}\right)\|f\|^{2} \\
& =\left(\nu+\frac{1-\nu}{1+|p|^{2}}\right)\left(\frac{1+|p|}{1-|p|}\right)\|f\|^{2},
\end{aligned}
$$

which, upon recalling that $f$ is any function in $H^{2}$, yields $\left\|C_{\varphi}\right\|^{2} \leq$ $\delta \frac{1+|p|}{1-|p|}$, where $\delta=\nu+\frac{1-\nu}{1+|p|^{2}}$ is $<1$ (because $p \neq 0$ ). Thus $\left\|C_{\varphi}\right\|<\sqrt{\frac{1+|p|}{1-|p|}}$, as desired. 


\section{Concluding Remarks}

6.1. The essential norm revisited. For each positive integer $n$, let $H_{n}^{2}$ be the subspace of $H^{2}$ consisting of functions that vanish at the origin to order $n$. It is shown in [18, page 393] that

$$
\left\|C_{\varphi}\right\|_{e}=\lim _{n \rightarrow \infty}\left\|\left.C_{\varphi}\right|_{H_{n}^{2}}\right\| .
$$

This, along with formula (15) and Theorem 4.1, implies that if $\varphi$ is not inner and $\varphi(0)=0$ then $\left\|\left.C_{\varphi}\right|_{H_{n}^{2}}\right\|$ must, from some point on, have norm $<1$. Theorem 5.2 shows that this reduction of norm happens right at the first step.

6.2. The essential spectral radius. In [15] Poggi-Corradini showed that if $\varphi$ is a holomorphic self-map of $\mathbb{U}$ that is not inner, with $\varphi(0)=0$ and $\varphi^{\prime}(0) \neq 0$, then the essential spectral radius of $C_{\varphi}$ is $<1$ (actually, he found a precise formula for the essential spectral radius in terms of the geometric properties of the Koenigs function of $\varphi$ ). Theorem 5.2 above shows that even if $\varphi^{\prime}(0)=0$ the essential spectral radius will still be $<1$, and provides yet another characterization of innerness:

If $\varphi$ fixes the origin and is not a rotation, then $\varphi$ is inner if and only if the essential spectral radius of $C_{\varphi}$ is 1.

For the (non-essential) spectral radius there is a version of this result that parallels Theorem 5.2:

$$
\text { If } \varphi(0)=0 \text { then } \varphi \text { is inner if and only if } r\left(\left.C_{\varphi}\right|_{H_{0}^{2}}\right)=1 \text {. }
$$

Indeed, one direction follows from the fact that the spectral radius of any operator is dominated by its norm, and the other from the fact, by the spectral radius formula, every isometry has spectral radius 1 .

6.3. A more general restriction theorem. There is also a restriction theorem for the norm of $C_{\varphi}$ when $\varphi(0)=p \neq 0$. Let $H_{p}^{2}$ be the subspace of $H^{2}$ consisting of functions that vanish at $p$. Then:

$$
\varphi \text { is inner if and only if }\left\|\left.C_{\varphi}\right|_{H_{p}^{2}}\right\|=\sqrt{\frac{1+|p|}{1-|p|}} .
$$

To see why this is so note that if the norm of $C_{\varphi}$ on $H_{p}^{2}$ is $\sqrt{\frac{1+|p|}{1-|p|}}$ then that is also its norm on all of $H^{2}$, whereupon Theorem 5.2 guarantees that $\varphi$ is inner. On the other hand, if $\varphi$ is inner, then we know from (1) that the norm of $C_{\varphi}$ (viewed as an operator on all of $H^{2}$ ) is $\sqrt{\frac{1+|p|}{1-|p|}}$. Because $\alpha_{p} \circ \varphi$ is again inner we have for every $g \in H^{2}$ :

$$
\left\|C_{\varphi}\left(\alpha_{p} \cdot g\right)\right\|=\left\|\left(\alpha_{p} \circ \varphi\right) \cdot C_{\varphi} g\right\|=\left\|C_{\varphi} g\right\|,
$$


and since $H_{p}^{2}=\alpha_{p} \cdot H^{2}$ this implies that

$$
\left\|\left.C_{\varphi}\right|_{H_{p}^{2}}\right\|=\left\|C_{\varphi}\right\|=\sqrt{\frac{1+|p|}{1-|p|}}
$$

as promised.

6.4. The numerical range. Valentin Matache [12] previously obtained a special case of Theorem 5.2, proving the inequality $\left\|\left.C_{\varphi}\right|_{H_{0}^{2}}\right\|<$ 1 for a sub-class of non-inner holomorphic self-maps $\varphi$ of $\mathbb{U}$ that fix the origin. Matache used his result to get information about the numerical range of the composition operators induced by these maps. Theorem 5.2 allows Matache's result to be extended to the full class of non-inner self-maps that fix the origin, as I now describe.

The numerical range of a bounded linear operator $T$ on a Hilbert space $\mathcal{H}$ is the set of complex numbers

$$
W(T)=\{\langle T x, x\rangle: x \in \mathcal{H},\|x\|=1\} .
$$

According to the Toeplitz-Hausdorff theorem (see [10, page 112] or [9, page 4]) $W(T)$ is convex, and it is not difficult to show that its closure $\bar{W}(T)$ contains the spectrum of $T$ ([9, $\S 1.2$, page 6$],[10$, Problem 214, page 115]). Thus, denoting the spectral radius of $T$ by $r(T)$, and the numerical radius by $w(T)$ (the supremum of the moduli of the points in the numerical range), we have:

$$
r(T) \leq w(T) \leq\|T\| .
$$

Now the Hilbert space $H^{2}$ decomposes into the orthogonal direct sum of the closed subspace $H_{0}^{2}$ and the subspace of constant functions. When $\varphi(0)=0$, these subspaces reduce $C_{\varphi}$, i.e., they are both $C_{\varphi^{-}}$ invariant. It is not difficult to check that in this case $W\left(C_{\varphi}\right)$ is the convex hull of $W\left(\left.C_{\varphi}\right|_{H_{0}^{2}}\right)$ and the singleton $\{1\}$. In particular if $\varphi$ fixes the origin and is not inner, then by Theorem 5.2 we have $\rho=$ $\left\|\left.C_{\varphi}\right|_{H_{0}^{2}}\right\|<1$, so $\bar{W}\left(\left.C_{\varphi}\right|_{H_{0}^{2}}\right) \subset \rho \overline{\mathbb{U}}$, hence $\bar{W}\left(C_{\varphi}\right)$ lies in the convex hull of $\rho \overline{\mathbb{U}}$ and $\{1\}$, and is, in particular, not $\overline{\mathbb{U}}$.

In the converse direction, if $\varphi$ is inner, fixes the origin, and is not a rotation, then $C_{\varphi}$ is a non-surjective isometry of $H^{2}$, so its spectrum is the closed unit disc, and therefore $\bar{W}\left(C_{\varphi}\right) \supset \overline{\mathbb{U}}$. But also $w\left(C_{\varphi}\right) \leq$ $\left\|C_{\varphi}\right\|=1$, so the opposite containment holds, hence $\bar{W}\left(C_{\varphi}\right)=\overline{\mathbb{U}}$. To summarize, if $\varphi(0)=0$ then:

(a) If $\varphi$ is not inner, then $\bar{W}\left(C_{\varphi}\right)$ is a proper subset of $\overline{\mathbb{U}}$; in fact, it is not a disc.

(b) If $\varphi$ is inner and not a rotation, then $\bar{W}\left(C_{\varphi}\right)=\overline{\mathbb{U}}$. 
If $\varphi$ is a rotation about the origin, then it is easy to check that $\bar{W}\left(C_{\varphi}\right)$ is the closed unit disc if the angle of rotation is an irrational multiple of $\pi$; otherwise it is a regular polygon inscribed in the unit circle.

It is not known if $\bar{W}\left(C_{\varphi}\right)$ is a disc whenever $\varphi(0) \neq 0$ and $\varphi$ is not an elliptic automorphism. The problem is that similar operators do not, in general, have the same the numerical range. Bourdon and I have established this circularity for non-elliptic conformal automorphisms of $\mathbb{U}$, and for elliptic ones whose multiplier is not a root of unity [1], but the general problem remains open and interesting.

\section{ACKNOWLEDGMENT}

I wish to thank Paul Bourdon of Washingon and Lee University for much valuable advice and encouragement during the preparation of this paper. In particular, Bourdon suggested the organization of $\S 5$, which greatly simplified my original proof of Theorem 5.2.

\section{REFERENCES}

[1] P. S. Bourdon and J. H. Shapiro, The numerical range of a composition operator, in preparation.

[2] P. S. Bourdon and J. H. Shapiro, Riesz composition operators, Pacific J. Math 181 (1997), 231-246.

[3] C. C. Cowen, Linear fractional composition operators on $H^{2}$, Integral Eqns. Op. Th. 11 (1988), 151-160.

[4] C. C. Cowen and B. D. MacCluer, Composition Operators on Spaces of Analytic Functions, CRC Press, Boca Raton, 1995.

[5] P. L. Duren, Theory of $H^{p}$ Spaces, Academic Press, New York, 1983.

[6] M. Essén, D. F. Shea, and C. S. Stanton, A value-distribution criterion for the class L Log L, and some related questions, Ann. Inst. Fourier (Grenoble) 35 (1985), 127-150.

[7] J. B. Garnett, Bounded Analytic Functions, Academic Press, New York, 1981.

[8] F. Jafari et al., editors, Studies on Composition Operators, Contemp. Math. Vol. 213, American Math. Soc. 1998.

[9] K. E. Gustafson and K. M. Rao, The Numerical Range, The Field of Values of Linear Operators and Matrices, Springer, New York, 1997.

[10] P. R. Halmos, A Hilbert Space Problem Book, Second Edition, Springer, New York 1982.

[11] J. E. Littlewood, On inequalities in the theory of functions, Proc. London Math. Soc. 23 (1925), 481-519.

[12] V. Matache, Numerical ranges of composition operators, preprint 1999.

[13] R. Nevanlinna, Analytic Functions, Springer-Verlag, New York, 1970.

[14] E. A. Nordgren, Composition operators, Canadian J. Math. 20 (1968), 442-449.

[15] P. Poggi-Corradini, The Hardy class of Koenigs maps, Michigan Math. J. 44 (1997), 495-507. 
[16] W. Rudin, A generalization of a theorem of Frostman, Math. Scand. 21 (1967), 136-173.

[17] W. Rudin, Real and Complex Analysis, 3rd edition, McGraw Hill, New York, 1987.

[18] J. H. Shapiro, The essential norm of a composition operator, Annals of Math. 125 (1987), 375-404.

[19] J. H. Shapiro, Composition Operators and Classical Function Theory, Springer-Verlag, New York, 1993.

[20] J. H. Shapiro, Recognizing an inner function by its distribution of values, unpublished manuscript 1999, available at the Web address: http://www.math.msu.edu/ shapiro/preprints/.

[21] C. S. Stanton, Riesz mass and growth problems for subharmonic functions, Thesis, Univ. of Wisconsin, Madison 1982.

Michigan State University, East LAnsing, MI 48824-1027, USA

E-mail address: shapiro@math.msu.edu 